\title{
Normalization of Naxos plakoglobin levels restores cardiac function in mice
}

\author{
Zhiwei Zhang, ${ }^{1,2}$ Matthew J. Stroud, ${ }^{1}$ Jianlin Zhang, ${ }^{1}$ Xi Fang, ${ }^{1}$ Kunfu Ouyang, ${ }^{3}$ Kensuke Kimura, ${ }^{1}$ Yongxin Mu, ${ }^{1}$ Nancy D. Dalton, ${ }^{1}$ \\ Yusu Gu, ${ }^{1}$ William H. Bradford, ${ }^{1}$ Kirk L. Peterson, ${ }^{1}$ Hongqiang Cheng, ${ }^{4}$ Xinmin Zhou, ${ }^{2}$ and Ju Chen ${ }^{1}$ \\ 'School of Medicine, UCSD, La Jolla, California, USA. 'Department of Cardiothoracic Surgery, The Second Xiangya Hospital, Central South University, Changsha, Hunan, China. ${ }^{3}$ School of Chemical Biology and \\ Biotechnology, Peking University, Shenzhen, China. ${ }^{4}$ Department of Pathology and Pathophysiology, Program in Molecular Cell Biology, Zhejiang University School of Medicine, Hangzhou, China.
}

\begin{abstract}
Arrhythmogenic cardiomyopathy $(\mathrm{AC})$ is associated with mutations in genes encoding intercalated disc proteins and ultimately results in sudden cardiac death. A subset of patients with $A C$ have the autosomal recessive cardiocutaneous disorder Naxos disease, which is caused by a 2-base pair deletion in the plakoglobin-encoding gene JUP that results in a truncated protein with reduced expression. In mice, cardiomyocyte-specific plakoglobin deficiency recapitulates many aspects of human $A C$, and overexpression of the truncated Naxos-associated plakoglobin also results in an AC-like phenotype; therefore, it is unclear whether Naxos disease results from loss or gain of function consequent to the plakoglobin mutation. Here, we generated 2 knockin mouse models in which endogenous Jup was engineered to express the Naxos-associated form of plakoglobin. In one model, Naxos plakoglobin bypassed the nonsense-mediated mRNA decay pathway, resulting in normal levels of the truncated plakoglobin. Moreover, restoration of Naxos plakoglobin to WT levels resulted in normal heart function. Together, these data indicate that a gain of function in the truncated form of the protein does not underlie the clinical phenotype of patients with Naxos disease and instead suggest that insufficiency of the truncated Naxos plakoglobin accounts for disease manifestation. Moreover, these results suggest that increasing levels of truncated or WT plakoglobin has potential as a therapeutic approach to Naxos disease.
\end{abstract}

\section{Introduction}

Arrhythmogenic cardiomyopathy (AC) (1-3) is observed in Naxos disease, which is caused by a 2-base pair deletion in the JUP gene encoding the intercalated disc (ICD) protein plakoglobin (1). This mutation causes a frameshift, resulting in a premature stop codon, and expression of a truncated plakoglobin lacking 56 residues from the $\mathrm{C}$ terminus. Naxos disease is autosomal recessive, suggesting that truncated plakoglobin is unable to function as its full-length counterpart. Studies to model Naxos disease have yielded different conclusions.

To investigate whether loss of plakoglobin in cardiomyocytes recapitulates Naxos cardiac disease, several mouse models have been generated. Ablation of plakoglobin in adult cardiomyocytes recapitulated many features of Naxos disease, including progressive cardiomyocyte loss, excessive inflammation, and fibrosis. Furthermore, desmosomal ultrastructure and cardiac function were affected in these mice (4). Ablation of plakoglobin in fetal cardiomyocytes resulted in mice with a more severe Naxos-like cardiac phenotype, with cardiac dysfunction, fibrosis, ventricular thinning, and spontaneous ventricular arrhythmias (5). These mouse models, and the recessive nature of Naxos disease, suggest that the disorder is governed by a loss of function (LOF) of plakoglobin.

Authorship note: Zhiwei Zhang and Matthew J. Stroud contributed equally to this work. Conflict of interest: The authors have declared that no conflict of interest exists. Submitted: December 5, 2014; Accepted: January 9, 2015.

Reference information: J Clin Invest. 2015;125(4):1708-1712. doi:10.1172/JCI80335.
In addition to LOF mouse models, gain-of-function (GOF) transgenic mice and zebrafish overexpressing the truncated plakoglobin have been generated $(6,7)$. In GOF models, many characteristic signs of Naxos cardiomyopathy are also observed, including fibrofatty replacement of cardiomyocytes. Overexpressed mutant plakoglobin, in vivo in cardiomyocytes $(6,7)$ or in cultured neonatal rat ventricular myocytes, localized to the nucleus $(6,7)$ and interfered with $\beta$-catenin signaling $(6,7)$. Currently, there are no data from cardiac tissue from patients with Naxos disease that address the extent to which the mutant plakoglobin protein is reduced or its subcellular localization changed.

To gain insight into mechanisms underlying Naxos disease, we used mouse genetics to generate 2 mouse models in which the Jup gene was modified to recapitulate the Naxos mutation. Mice that bypassed nonsense-mediated decay to express truncated plakoglobin at levels comparable to those of full-length WT protein did not display any phenotype characteristic of Naxos disease. Our data demonstrated that GOF of the mutant protein does not underlie the clinical phenotype of patients with Naxos disease, leaving the alternate explanation that LOF of Naxos protein accounts for disease manifestation. An important implication of our findings is that a viable therapeutic approach to Naxos disease will be to increase levels of truncated or WT plakoglobin protein.

\section{Results and Discussion}

A 2-base pair deletion in both alleles of the JUP gene encoding plakoglobin causes Naxos disease in humans (1). To investigate this disease in mice and to exactly mimic the mutation found in 

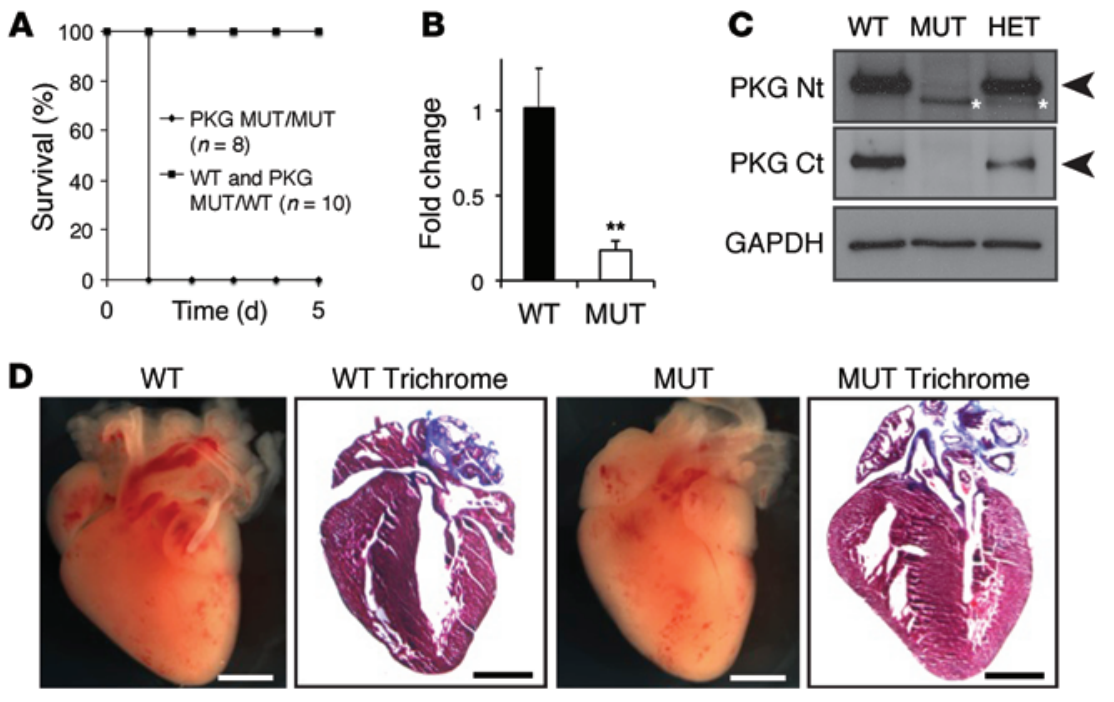

$\mathbf{E}$
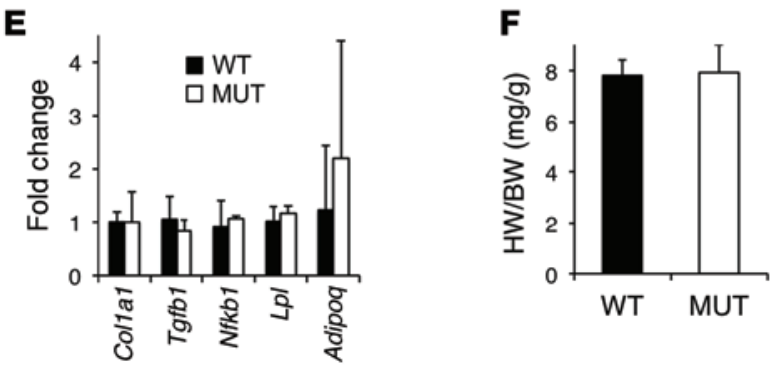

Figure 1. Characterization of the OriNax knockin mouse. (A) Kaplan-Meier survival curves of WT or OriNax (MUT) mice. Note that all OriNax pups died at PD1, while none of WT littermates did. PKG, plakoglobin. (B) Quantification of mRNA levels of plakoglobin relative to Gapdh levels. Note significant downregulation in the mutant allele. $n=6$ mice per genotype. (C) Western blot of WT, heterozygote (HET), and homozygote OriNax mice with $\mathrm{N}$-terminal (PKC Nt) and C-terminal (PKC Ct) antibodies for plakoglobin. CAPDH served as a loading control. Note reduced molecular weight (MW) and amount of plakoglobin in OriNax and HET mice (white asterisks). Arrowheads denote predicted MW of plakoglobin. $n=6$ mice per genotype. (D) Macroscopic views of Masson's Trichrome-stained hearts isolated from WT and OriNax PD1 mice. Note lack of gross morphological defects or fibrosis in hearts from OriNax mice compared with WT littermates. Scale bar: $1 \mathrm{~mm} . n=6$ mice per genotype. (E) qRT-PCR analysis of indicated genes compared with Gapdh levels. Note that no changes of collagen 1A, Tgfb1, Nfkb, lipoprotein lipase, and adiponectin were observed between WT and OriNax hearts. $n=6$ mice per genotype. (F) Heart weight/body weight ratios (HW/BW) were calculated for WT and OriNax mice. $n=6$ mice per genotype. Data represent mean $\pm S E M$; ${ }^{* *} P<0.01$ according to 2 -tailed Student's $t$ test. human patients with Naxos disease, we generated a mouse line called original Naxos (OriNax), in which 2 base pairs were deleted to result in a frameshift and premature stop codon (Supplemental Figure 1, A-C; supplemental material available online with this article; doi:10.1172/JCI80335DS1).

OriNax mice were born at expected Mendelian ratios $(24.2 \%$ OriNax mice compared with $25 \%$ expected from 190 pups from heterozygote crosses) but died on postnatal day 1 (PD1) (Figure 1A). Plakoglobin mRNA levels were significantly downregulated in OriNax mice compared with those in WT littermates (Figure 1B). This was because the frameshift mutation introduced in exon 11 of the Jup gene results in a premature termination codon in exon 12 that is recognized by a translation-coupled surveillance pathway termed nonsense-mediated RNA decay. This pathway leads to the specific degradation of plakoglobin mRNA (8). In accordance with the lower mRNA levels, plakoglobin protein levels were markedly downregulated in OriNax mice compared with those in WT littermates (Figure 1C).

Masson's Trichrome staining revealed no loss of cardiomyocytes, fibrosis, thinning of the ventricles, and fatty deposits in PD1 hearts from OriNax mutants when compared with littermate controls (Figure 1D). Consistent with the absence of fatty deposits, we observed no changes in the levels of proadipogenic genes, such as lipoprotein lipase or adiponectin (Figure 1E). Furthermore, we observed no changes in heart weight/body weight ratios (Figure $1 \mathrm{~F}$ ) or collagen $1 \mathrm{~A}$ or proinflammatory genes $\mathrm{Tg} f b 1$ or $\mathrm{Nfkb}$ (Figure 1E). Global plakoglobin knockout mice die from cardiac defects at around embryonic day $13(9,10)$. Despite OriNax mice having low levels of plakoglobin, they appear to have a sufficient amount for normal cardiac development. However, the skin of OriNax mice was more fragile and wrinkled than that of WT controls. Furthermore, Masson's Trichrome staining of skin revealed that the epidermis of OriNax mice was thinner and looser than that of their WT littermates (Supplemental Figure 1D).

Plakoglobin LOF models exhibit alterations in levels of ICD proteins in cardiac tissue $(4,5)$; therefore, we investigated levels of ICD proteins in cardiac tissue from OriNax mice. Levels of adherens junction components $\alpha$-catenin and cadherins, as well as gap junction and costameric proteins, were unaffected in OriNax mice when compared with those of their WT littermates (Supplemental Figure 2, A-C). Levels of most of the desmosomal components, desmoplakin (DSP), desmocollin 2 (DSC2), and desmoglein 2 (DSG2), were markedly downregulated (Supplemental Figure 2, D-F), although plakophilin 2 (PKP2) was unaffected. Plakoglobin shares homology with $\beta$-catenin, and it has been proposed that they may be able to functionally compensate for one another $(11,12)$. Similar to observations with plakoglobin LOF models, we observed elevated $\beta$-catenin levels in OriNax mice (Supplemental Figure 2, A and G). It should be noted that the low intensity immunofluorescence of ICD components at PD1 is to be expected, because the ICD matures postnatally (13).

Overall, OriNax mice died soon after birth and did not display characteristic hallmarks of AC. The lack of cardiac phenotype is not too surprising when understood in the context of the progression of Naxos disease, in which cardiac phenotype is age dependent (2). OriNax mutant perinatal lethality has also been observed in $50 \%$ of mice that are hypomorphic for WT plakoglobin, expressing approximately $40 \%$ of normal levels of WT plakoglobin (14). Perinatal lethality has not been reported for patients with Naxos disease. Reasons for this discrepancy are not clear but may reflect 
A

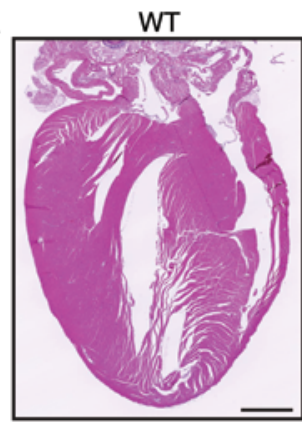

B

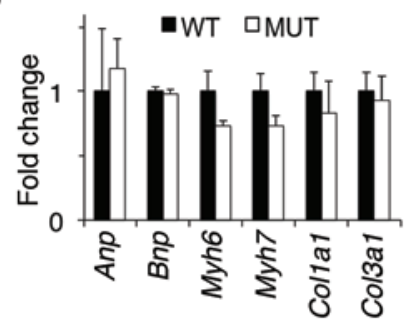

WT

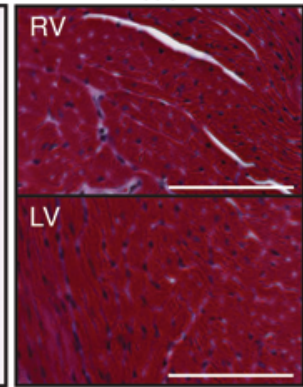

C

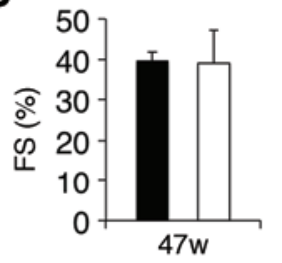

E

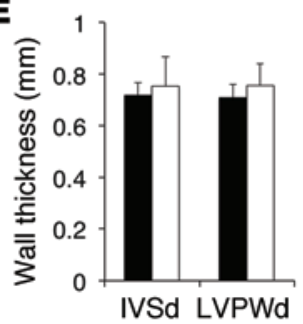

MUT

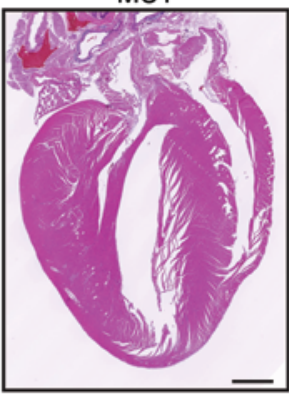

D

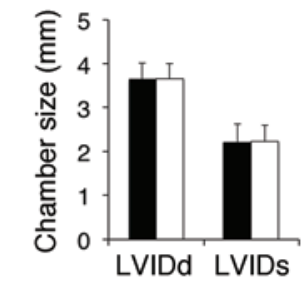

$\mathbf{F}$
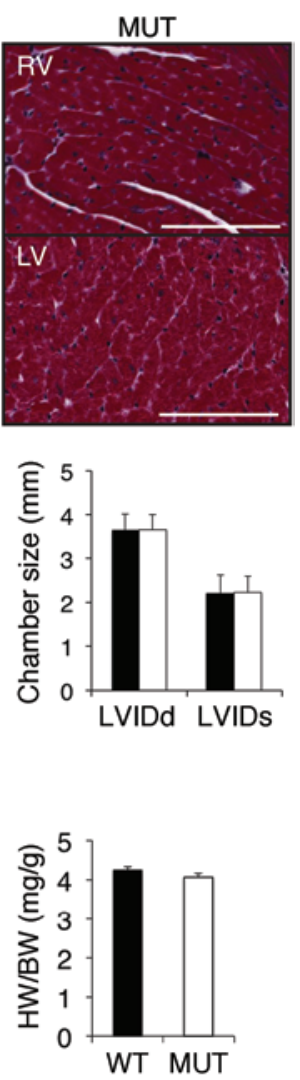

Figure 2. No overt changes in cardiac remodeling, function, hypertrophy, or the fetal gene program were observed in the FuseNax mice. (A) Fourchamber views of Masson's Trichrome-stained hearts taken from 4-month-old WT and FuseNax (MUT) mice. Scale bar: 1 mm. Higher-magnification images of right ventricles (RV) and left ventricles (LV) are shown to the right of respective 4-chamber views. Note no significant morphological changes or increase in fibrosis. $n=3$ mice per genotype. Scale bar: $100 \mu \mathrm{m}$. (B) qRT-PCR analysis of the indicated genes showing the fold change relative to $18 \mathrm{~S}$ from 10-month-old mice. Note that expression of the fetal gene program (Anp, Bnp, aMHC, $\beta M H C$ ) and fibrotic markers (collagen 1A1, collagen 3A1) were unchanged in FuseNax mice compared with those in WT mice. Data represent mean $\pm S E M ; n=8$ mice per genotype. (C-E) Fractional shortening (FS) was calculated, and chamber size and wall thicknesses were measured in 47-week-old mice using echocardiography. Note that no changes were observed in heart function. Data represent mean $\pm S D, n=16$ mice per genotype. LVIDd, left ventricle internal diameter, diastole; LVIDs, left ventricular internal diameter, systole; IVSd, interventricular septum diameter; LVPWd, LV posterior wall, diastole. (F) Heart weight/body weight ratios were unaffected in the FuseNax mice. Data represent mean \pm SEM; $n=16$ mice per genotype. Two-tailed Student's $t$ test was performed on data.

the production of different levels of plakoglobin protein from the mutant gene in human patients versus mouse models or divergent requirements for the amount of plakoglobin in the two species.

OriNax mice expressed truncated plakoglobin at very low levels, making it hard to conclude whether observed phenotypes were owing to low expression levels or to expression of the truncated form of the protein. In an attempt to bypass nonsense-mediated RNA decay and increase the amount of the truncated plakoglobin, we generated a new mouse line called fused exon Naxos (FuseNax). These mice have the last 5 exons of the Jup gene fused together (Supplemental Figure 3, A and B) and expressed the truncated plakoglobin at the same level as full-length plakoglobin (see below and Supplemental Figure 3C).

Pathological cardiac remodeling and cardiac dysfunction were reported in transgenic mice and zebrafish overexpressing truncated plakoglobin, resulting in premature death $(6,7)$. FuseNax mice were born at expected Mendelian ratios, surviving with no overt morphological phenotype.

Masson's Trichrome staining of heart sections revealed no gross morphological defects, changes in fibrosis, or cardiomyo- cyte loss in FuseNax mice (Figure 2A). Cardiac remodeling is often accompanied by activation of the fetal gene program (15). Consistent with our histological data, we found no changes in the fetal gene program (Figure 2B). Furthermore, we observed no differences in cardiac function, heart weight/body weight ratio, chamber size, or wall thickness between FuseNax mice and their WT littermates at 47 weeks of age and older (Figure 2, C-F, and data not shown). In contrast to OriNax mice, no skin or hair abnormalities were observed in FuseNax mice (data not shown).

Plakoglobin plays a fundamental role at the ICD; therefore, we sought to understand whether expression of truncated plakoglobin would affect other ICD proteins. Both subcellular localization and expression levels of $\beta$-catenin and connexin 43 appeared normal in myocardium of FuseNax mice when compared with WT littermates (Figure 3, A-D). Furthermore, the majority of ICD proteins were unaffected in FuseNax mice (Figure 3, C-E). However, levels of DSC2, a desmosomal protein that interacts with plakoglobin (16), were reduced to $40 \%$ of those of WT mice, as measured by densitometry of the Western blot (Figure 3E). 
A

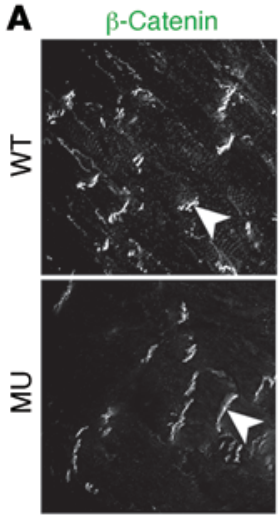

B
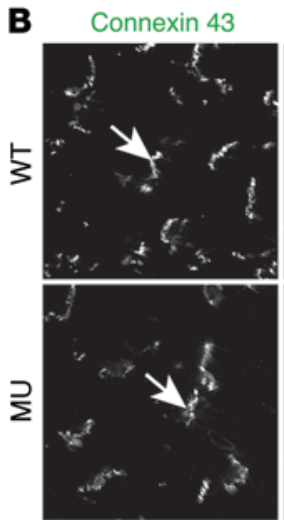

G

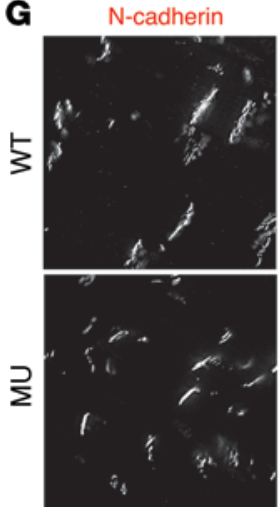

DAPI

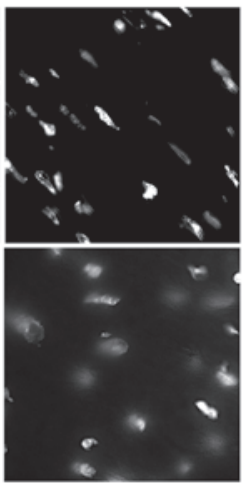

DAPI
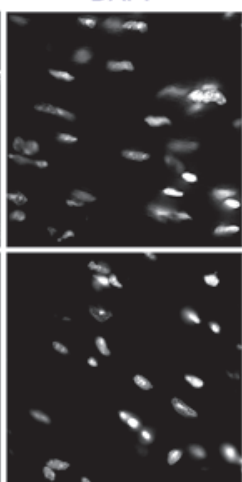

PKG
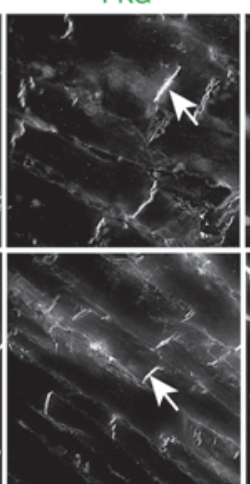

$\alpha$-Actinin

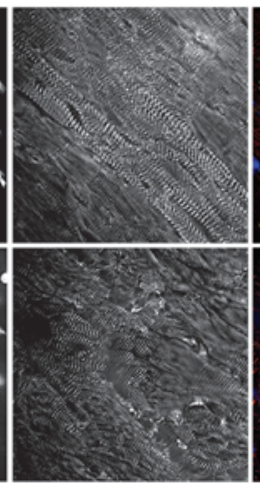

$\alpha$-Actinin

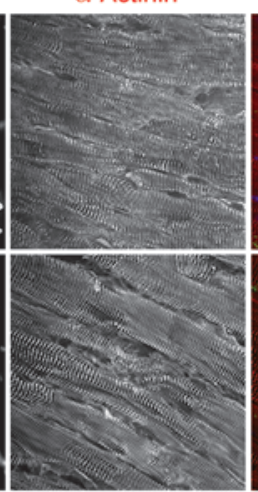

DAPI

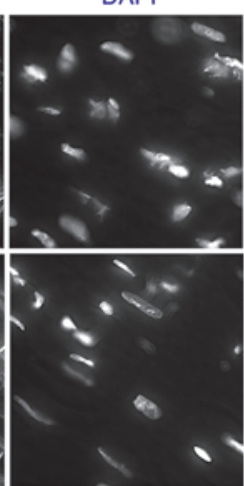

Merge
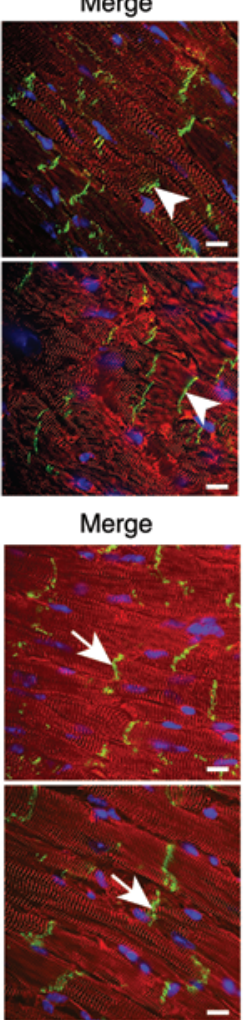

Merge

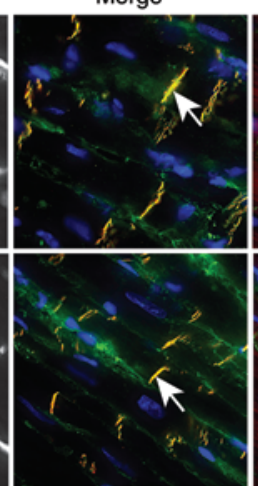

C

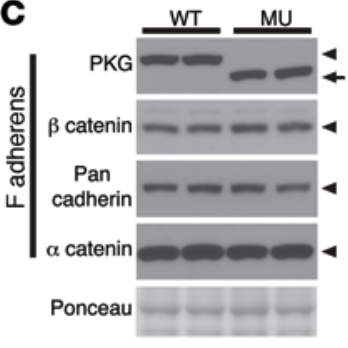

D

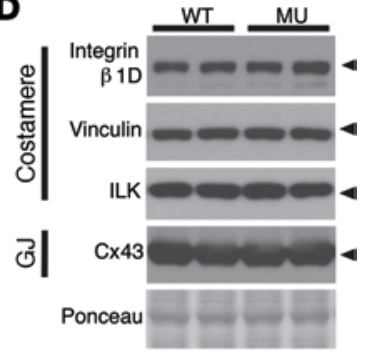

E

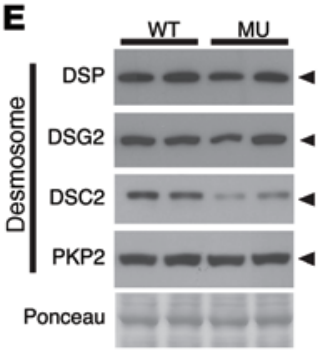

F
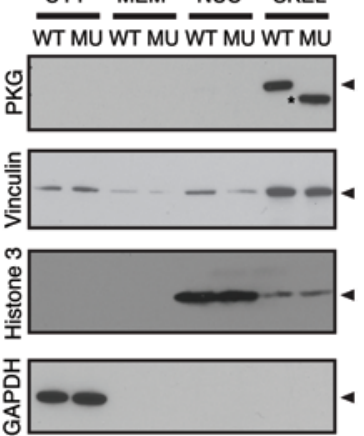

H Actin merge

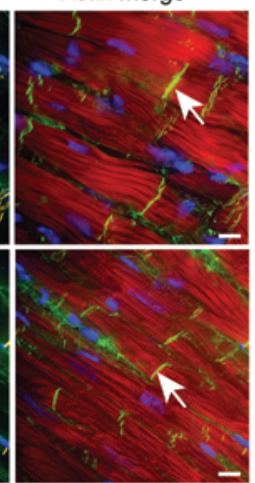

WT $\square \mathrm{MU}$

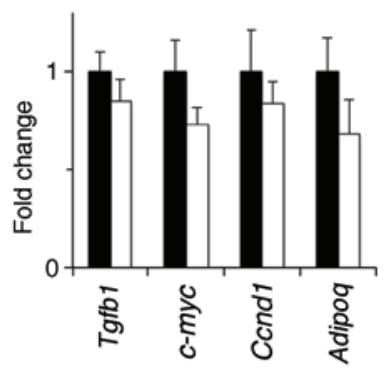

Figure 3. ICD protein expression levels and localizations were unaffected in FuseNax mice. (A and B) Immunofluorescence of $\beta$-catenin and connexin 43 (Cx43), respectively, in mouse myocardium. Note similar localization and levels of both $\beta$-catenin (white arrowheads) and Cx43 (white arrows) at ICDs between WT and FuseNax (MU) mice. $n=3$ mice per genotype. (C-E) Western blots of the indicated ICD proteins. Arrowheads show the predicted MW, and the arrow shows the predicted MW of mutant plakoglobin (PKC). Note that most protein levels are unaffected in the FuseNax mouse hearts. [x43, connexin 43; G], gap junction. $n=6$ mice per genotype. (F) Subcellular fractionation experiments with WT and FuseNax mouse hearts. Note that plakoglobin in both WT and FuseNax (asterisk) is in cytoskeletal (SKEL) fractions in heart tissue. As controls for fractionation, GAPDH is in the cytoplasm (CYT), histone 3 is in the nuclear fraction (NUC), and vinculin is predominantly in the cytoskeletal fraction. MEM, membrane fraction. $n=6$ mice per genotype. (C) Immunofluorescence of mouse myocardium using antibodies generated against plakoglobin. Note localization of plakoglobin (green) to ICD (white arrows) and colocalization with $\mathrm{N}$-cadherin. $n=3$ mice per genotype. (H) qRT-PCR analysis of the indicated genes, showing the fold change relative to $18 \mathrm{~S}$ from 10 -month-old mice. Note that expression of genes downstream of the Wnt/ $\beta$-catenin pathway was unaffected (c-myc, cyclin D1) as was the profibrotic marker TCF- $\beta$ (Tgfb1) and the adipogenic gene, adiponectin. $n=8$ mice per genotype. Data represent mean \pm SEM. Two-tailed Student's $t$ test was performed on data. Scale bar: $10 \mu \mathrm{m}$.

It has been shown that an overexpressed truncated Naxos plakoglobin protein translocates to the nucleus and interferes with $\beta$-catenin signaling $(6,7)$. To examine whether this nuclear localization occurred in mice expressing truncated plakoglobin under the endogenous promoter, we performed experiments using 2 antibodies generated against different epitopes at the $\mathrm{N}$ terminus of plakoglobin. We found no evidence of mutant protein localiz- ing to the nucleus, using either immunofluorescence or biochemical subcellular fractionation approaches (Figure 3, F and G, and Supplemental Figure 3D). Furthermore, genes downstream of $\beta$-catenin were unaffected in FuseNax mice (Figure 3H).

Given that FuseNax mice showed no impairment of cardiac contractile function at 11 months of age, we wanted to investigate whether they would exhibit impairment in cardiac contrac- 
tile function in response to $\beta$-adrenergic stimulation. Accordingly, 9-week-old mice were treated with increasing amounts of dobutamine and subjected to hemodynamic analyses. Results demonstrated that FuseNax mice responded similarly to WT littermates, with no impairment in contractile function in response to $\beta$-adrenergic stimulation (Supplemental Figure $4, \mathrm{~A}-\mathrm{F}$ ).

To investigate whether FuseNax mice displayed arrhythmias, we performed telemetry recordings over a 48 -hour period. We found no changes in heart rate between FuseNax and WT littermates (Supplemental Figure 4, G and H). Furthermore, QRS complex duration and PR interval were unaffected (Supplemental Figure 4I). In addition, no ventricular tachycardia was detected.

Previous approaches to understand molecular mechanisms underlying AC in Naxos disease have involved conditional knockout approaches and transgenic mouse and zebrafish models in which plakoglobin is overexpressed $(6,7)$. Here, we specifically generated 2 knockin mouse models in which the Jup gene encoding plakoglobin was modified to mimic the Naxos mutation. Our initial OriNax model exhibited perinatal lethality, as observed by Ruiz et al. in a mouse model with significantly reduced levels of WT plakoglobin (9). In FuseNax mice, in which the truncated Naxos plakoglobin is expressed at levels equivalent to the WT protein, we found no impairment of cardiac function, cardiac hypertrophy, or chamber dilation and no evidence for increased fibrosis, fibrofatty replacement, or myofibrillar disarray. These observations demonstrated that WT levels of truncated Naxos plakoglobin in FuseNax mice were sufficient to maintain cardiac function.

It should be pointed out, however, that although hearts appeared to be functionally normal, DSC2 protein levels were consistently reduced in FuseNax hearts, implying that the $\mathrm{C}$ terminus of WT plakoglobin may stabilize DSC2 at the ICD, and the truncated protein may not possess the full function of WT plakoglobin.

Overall, our results showed that restoration of truncated Naxos plakoglobin to WT levels resulted in normal cardiac function. These data demonstrate that GOF of mutant Naxos protein does not underlie the clinical phenotype of patients with Naxos disease, leaving the alternate explanation that insufficiency of Naxos protein accounts for disease manifestation. Importantly, our results suggest that increasing levels of truncated plakoglobin or WT plakoglobin protein would be beneficial for treatment of patients with Naxos disease. One approach to this would be to use antisense technology to specifically block nonsense-mediated decay of mutant plakoglobin mRNA, enabling expression of the truncated protein at increased levels (17).

\section{Methods}

Statistics. Data are presented as mean \pm SEM unless indicated otherwise. We used 2-tailed Student's $t$ test for comparisons among groups. Analysis was performed using Microsoft Excel software. $P$ values of less than 0.05 were considered significant.

Study approval. All animal procedures were approved by the UCSD Animal Care and Use Committee. UCSD has an Animal Welfare Assurance (A3033-01) on file with the Office of Laboratory Animal Welfare and is fully accredited by AAALAC International.

See the Supplemental Methods for additional details; see Supplemental Table 1 for details regarding antibodies and Supplemental Table 2 for primers used for qRT-PCR.

\section{Acknowledgments}

M.J. Stroud was supported by an American Heart Association postdoctoral fellowship (13POST17060120). J. Chen was funded by grants from National Heart, Lung, and Blood Institute and is the American Heart Association Endowed Chair. Microscopy work was performed at the UCSD Neuroscience Microscopy Shared Facility and was supported by the NIH (grant P30 NS047101).

Address correspondence to: Ju Chen, UCSD School of Medicine, 9500 Gilman Drive, La Jolla, California 92093-0613, USA. Phone: 858.822.4276; E-mail: juchen@ucsd.edu.
1. McKoy G, et al. Identification of a deletion in plakoglobin in arrhythmogenic right ventricular cardiomyopathy with palmoplantar keratoderma and woolly hair (Naxos disease). Lancet. 2000;355(9221):2119-2124.

2. Kaplan SR, et al. Remodeling of myocyte gap junctions in arrhythmogenic right ventricular cardiomyopathy due to a deletion in plakoglobin (Naxos disease). Heart Rhythm. 2004;1(1):3-11.

3. Protonotarios $\mathrm{N}$, et al. Cardiac abnormalities in familial palmoplantar keratosis. Br Heart J. 1986;56(4):321-326.

4. Li J, Swope D, Raess N, Cheng L, Muller EJ, Radice GL. Cardiac tissue-restricted deletion of plakoglobin results in progressive cardiomyopathy and activation of $\beta$-catenin signaling. Mol Cell Biol. 2011;31(6):1134-1144.

5 . Li D, et al. Restrictive loss of plakoglobin in cardiomyocytes leads to arrhythmogenic cardiomyopathy. Hum Mol Genet. 2011;20(23):4582-4596.

6. Lombardi R, da Graca Cabreira-Hansen M, Bell A, Fromm RR, Willerson JT, Marian AJ. Nuclear plakoglobin is essential for differentiation of cardiac progenitor cells to adipocytes in arrhythmogenic right ventricular cardiomyopathy. Circ Res. 2011;109(12):1342-1353.

7. Asimaki A, et al. Identification of a new modulator of the intercalated disc in a zebrafish model of arrhythmogenic cardiomyopathy. Sci Transl Med. 2014;6(240):240ra74.

8. Frischmeyer PA, Dietz HC. Nonsense-mediated mRNA decay in health and disease. Hum Mol Genet. 1999;8(10):1893-1900.

9. Ruiz P, et al. Targeted mutation of plakoglobin in mice reveals essential functions of desmosomes in the embryonic heart. J Cell Biol. 1996;135(1):215-225.

10. Bierkamp C, McLaughlin KJ, Schwarz H, Huber O, Kemler R. Embryonic heart and skin defects in mice lacking plakoglobin. Dev Biol. 1996;180(2):780-785.

11. Swope D, Cheng L, Gao E, Li J, Radice GL. Loss of cadherin-binding proteins $\beta$-catenin and plakoglobin in the heart leads to gap junction remodeling and arrhythmogenesis. Mol Cell Biol. 2012;32(6):1056-1067.
12. Swope D, Li J, Radice GL. Beyond cell adhesion: the role of armadillo proteins in the heart. Cell Signal. 2013;25(1):93-100.

13. Hirschy A, Schatzmann F, Ehler E, Perriard JC. Establishment of cardiac cytoarchitecture in the developing mouse heart. Dev Biol. 2006;289(2):430-441.

14. Swope D, Li J, Muller EJ, Radice GL. Analysis of a Jup hypomorphic allele reveals a critical threshold for postnatal viability. Genesis. 2012;50(10):717-727.

15. Heineke J, Molkentin JD. Regulation of cardiac hypertrophy by intracellular signalling pathways. Nat Rev Mol Cell Biol. 2006;7(8):589-600.

16. Chitaev NA, Leube RE, Troyanovsky RB, Eshkind LG, Franke WW, Troyanovsky SM. The binding of plakoglobin to desmosomal cadherins: patterns of binding sites and topogenic potential. J Cell Biol. 1996;133(2):359-369.

17. Kole R, Krainer AR, Altman S. RNA therapeutics: beyond RNA interference and antisense oligonucleotides. Nat Rev Drug Discov. 2012;11(2):125-140. 